\title{
ASYMPTOTIC FORMULAS AND INEQUALITIES FOR GAMMA FUNCTION IN TERMS OF TRI-GAMMA FUNCTION
}

\author{
CRISTINEL MORTICI AND FENG QI
}

\begin{abstract}
In the paper, the authors establish some asymptotic formulas and double inequalities for the factorial $n$ ! and the gamma function $\Gamma$ in terms of the tri-gamma function $\psi^{\prime}$.
\end{abstract}

\section{INTRODUCTION AND MOTIVATION}

We recall that the classical Euler's gamma function may be defined by

$$
\Gamma(z)=\int_{0}^{\infty} t^{z-1} e^{-t} \mathrm{~d} t
$$

for $\Re(z)>0$, that the logarithmic derivative of $\Gamma(x)$ is called psi or di-gamma function and denoted by

$$
\psi(x)=\frac{\mathrm{d}}{\mathrm{d} x} \ln \Gamma(x)=\frac{\Gamma^{\prime}(x)}{\Gamma(x)}
$$

for $x>0$, that the derivatives $\psi^{\prime}(x)$ and $\psi^{\prime \prime}(x)$ for $x>0$ are respectively called tri-gamma and tetra-gamma functions, and that the derivatives $\psi^{(i)}(x)$ for $i \in \mathbb{N}$ and $x>0$ are called polygamma functions.

We also recall from [3, Chapter XIII] and [13, Chapter IV] that a function $f(x)$ is said to be completely monotonic on an interval $I$ if it has derivatives of all orders on $I$ and satisfies $0 \leq(-1)^{n} f^{(n)}(x)<\infty$ for $x \in I$ and all integers $n \geq 0$. The class of completely monotonic functions may be characterized by the celebrated Bernstein-Widder Theorem [13, p. 160, Theorem 12a] which reads that a necessary and sufficient condition that $f(x)$ should be completely monotonic in $0 \leq x<\infty$ is that

$$
f(x)=\int_{0}^{\infty} e^{-x t} \mathrm{~d} \alpha(t)
$$

where $\alpha(t)$ is bounded and non-decreasing and the integral converges for $0 \leq x<\infty$.

In [12, Theorem 2.1], it was proved that the function

$$
F_{\alpha}(x)=\ln \Gamma(x+1)-x \ln x+x-\frac{1}{2} \ln x-\frac{1}{2} \ln (2 \pi)-\frac{1}{12} \psi^{\prime}(x+\alpha)
$$

is completely monotonic on $(0, \infty)$ if and only if $\alpha \geq \frac{1}{2}$ and that the function $-F_{\alpha}(x)$ is completely monotonic on $(0, \infty)$ if and only if $\alpha=0$. Consequently, the double inequality

$$
\frac{x^{x}}{e^{x}} \sqrt{2 \pi x} \exp \left(\frac{1}{12} \psi^{\prime}\left(x+\frac{1}{2}\right)\right)<\Gamma(x+1)<\frac{x^{x}}{e^{x}} \sqrt{2 \pi x} \exp \left(\frac{1}{12} \psi^{\prime}(x)\right)
$$

was derived in [12, Corollary 2.1]. These results were also established in [5] and its preprint [6] independently from a different origin and by a different motivation. For some more information on bounding the gamma function $\Gamma$, please refer to the

2010 Mathematics Subject Classification. 26D15; 33B15; 41A10.

Key words and phrases. asymptotic formulas; inequalities; gamma function; tri-gamma function. This paper was typeset using $\mathcal{A M}_{\mathcal{M}}$-IATEX. 
newly published paper [10], the survey articles [7, 8, 9], and plenty of references collected therein.

The goal of this paper is to discover best asymptotic formulas and double inequalities for the factorial $n !=\Gamma(n+1)$ and the gamma function $\Gamma(x)$ in terms of the tri-gamma function $\psi^{\prime}\left(x+\frac{1}{2}\right)$. These results have something to do with the function $F_{\alpha}(x)$ and the double inequality (1.5).

\section{An ASYMPtotic FORMUla AND A DOUble INEQUALITY FOR $n$ !}

In this section, we establish a best asymptotic formula and a double inequality for the factorial $n !=\Gamma(n+1)$ in terms of the tri-gamma function $\psi^{\prime}\left(x+\frac{1}{2}\right)$.

Theorem 2.1. As $n \rightarrow \infty$, the asymptotic formula

$$
n ! \sim \frac{n^{n}}{e^{n}} \sqrt{2 \pi n} \exp \left(\frac{1}{12} \psi^{\prime}\left(n+\frac{1}{2}\right)\right)
$$

is the most accurate one among all approximations of the form

$$
n ! \sim \frac{n^{n}}{e^{n}} \sqrt{2 \pi n} \exp \left(\frac{1}{12} \psi^{\prime}(n+a)\right)
$$

where $a \in \mathbb{R}$.

Proof. For $n \geq 1$, define a sequence $w_{n}$ by

$$
n !=\Gamma(n+1)=\sqrt{2 \pi} n^{n+1 / 2} e^{-n} \exp \left(\frac{1}{12} \psi^{\prime}(n+a)\right) \exp w_{n} .
$$

Taking into account

$$
\psi^{(k)}(z+1)=\psi^{(k)}(z)+(-1)^{k} \frac{k !}{z^{k+1}}
$$

for $k=1$, see [1, p. 260, 6.4.6], yields

$$
w_{n+1}-w_{n}=1+\ln (n+1)-\left(n+\frac{3}{2}\right) \ln (n+1)+\left(n+\frac{1}{2}\right) \ln n+\frac{1}{12(n+a)^{2}}
$$

and

$$
w_{n+1}-w_{n}=\left(-\frac{1}{6} a+\frac{1}{12}\right) \frac{1}{n^{3}}+\left(\frac{1}{4} a^{2}-\frac{3}{40}\right) \frac{1}{n^{4}}+O\left(\frac{1}{n^{5}}\right) .
$$

Hence, we have

$$
\lim _{n \rightarrow \infty}\left\{n^{3}\left[w_{n+1}-w_{n}\right]\right\}=\frac{1}{12}-\frac{1}{6} a .
$$

Lemma 1.1 in $[4,11]$ states that if the sequence $\left\{\omega_{n}: n \in \mathbb{N}\right\}$ converges to 0 and

$$
\lim _{n \rightarrow \infty} n^{k}\left(\omega_{n}-\omega_{n+1}\right)=\ell \in \mathbb{R}
$$

for $k>1$, then

$$
\lim _{n \rightarrow \infty} n^{k-1} \omega_{n}=\frac{\ell}{k-1} .
$$

Consequently, the sequence $w_{n}$ converges fastest only if $a=\frac{1}{2}$.

Theorem 2.2. For every integer $n \geq 1$, we have

$$
\exp \left(\frac{1}{240 n^{3}}-\frac{11}{6720 n^{5}}\right)<\frac{e^{n} n !}{n^{n} \sqrt{2 \pi n} \exp \left(\frac{1}{12} \psi^{\prime}\left(n+\frac{1}{2}\right)\right)}<\exp \frac{1}{240 n^{3}} .
$$


Proof. The double inequality (2.6) may be rewritten as

$$
f(n)=\ln \Gamma(n+1)-\left(n+\frac{1}{2}\right) \ln n+n-\frac{1}{2} \ln (2 \pi)-\frac{1}{12} \psi^{\prime}\left(n+\frac{1}{2}\right)-\frac{1}{240 n^{3}} \leq 0
$$

and

$$
\begin{aligned}
g(n)=\ln \Gamma(n+1)-\left(n+\frac{1}{2}\right) \ln n & +n-\frac{1}{2} \ln (2 \pi) \\
& -\frac{1}{12} \psi^{\prime}\left(n+\frac{1}{2}\right)-\frac{1}{240 n^{3}}+\frac{11}{6720 n^{5}} \geq 0 .
\end{aligned}
$$

Employing the recurrence formula (2.3) applied to $k=1$ and straightforwardly computing reveal that $f(n+1)-f(n)=u(n)$ and $g(n+1)-g(n)=v(n)$, where

$$
\begin{aligned}
u(x)= & 1+\ln (x+1)-\left(x+\frac{3}{2}\right) \ln (x+1)+\left(x+\frac{1}{2}\right) \ln x \\
& +\frac{1}{12\left(x+\frac{1}{2}\right)^{2}}-\frac{1}{240(x+1)^{3}}+\frac{1}{240 x^{3}}
\end{aligned}
$$

and

$$
\begin{aligned}
v(x)= & 1+\ln (x+1)-\left(x+\frac{3}{2}\right) \ln (x+1)+\left(x+\frac{1}{2}\right) \ln x+\frac{1}{12\left(x+\frac{1}{2}\right)^{2}} \\
& -\frac{1}{240(x+1)^{3}}+\frac{1}{240 x^{3}}+\frac{11}{6720(x+1)^{5}}-\frac{11}{6720 x^{5}} .
\end{aligned}
$$

It is not difficult to verify that

$$
u^{\prime \prime}(x)=\frac{13 x+74 x^{2}+232 x^{3}+391 x^{4}+330 x^{5}+110 x^{6}+1}{20 x^{5}(x+1)^{5}(2 x+1)^{4}}>0
$$

and

where

$$
v^{\prime \prime}(x)=-\frac{Q(x)}{1120 x^{7}(x+1)^{7}(2 x+1)^{4}}<0
$$

$$
\begin{aligned}
Q(x)= & 825 x+5499 x^{2}+21325 x^{3}+52589 x^{4} \\
& +83867 x^{5}+83881 x^{6}+47936 x^{7}+11984 x^{8}+55 .
\end{aligned}
$$

This shows that $u(x)$ is strictly convex and $v(x)$ is strictly concave on $(0, \infty)$. Further considering $\lim _{x \rightarrow \infty} u(x)=\lim _{x \rightarrow \infty} v(x)=0$, we obtain that $u(x)>0$ and $v(x)<0$ on $(0, \infty)$. Consequently, the sequence $f(n)$ is strictly increasing and $g(n)$ is strictly decreasing while they both converge to 0 . As a result, we conclude that $f(n)<0$ and $g(n)>0$ for every integer $n \geq 1$. The proof of Theorem 2.2 is complete.

\section{An ASYMPtotic SERIES AND A DOUBLE INEQUALITY FOR $\Gamma$}

We now discover an asymptotic series and a double inequality for the gamma function $\Gamma(x)$ in terms of the tri-gamma function $\psi^{\prime}\left(x+\frac{1}{2}\right)$.

Theorem 3.1. As $x \rightarrow \infty$, we have

$$
\begin{aligned}
\Gamma(x+1) \sim \sqrt{2 \pi} x^{x+1 / 2} \exp ( & \frac{1}{12} \psi^{\prime}\left(x+\frac{1}{2}\right)-x+\frac{1}{240} \frac{1}{x^{3}} \\
& \left.-\frac{11}{6720} \frac{1}{x^{5}}+\frac{107}{80640} \frac{1}{x^{7}}-\frac{2911}{1520640} \frac{1}{x^{9}}+\cdots\right) .
\end{aligned}
$$


Proof. Motivated by the inequality (2.6), we now consider a new function $h(x)$ defined by

$$
\Gamma(x+1)=\sqrt{2 \pi} x^{x+1 / 2} e^{-x} \exp \left(\frac{1}{12} \psi^{\prime}\left(x+\frac{1}{2}\right)\right) \exp h(x),
$$

that is,

$$
h(x)=\left[\ln \Gamma(x+1)-\left(x+\frac{1}{2}\right) \ln x+x-\ln \sqrt{2 \pi}\right]-\frac{1}{12} \psi^{\prime}\left(x+\frac{1}{2}\right) .
$$

Using the formulas

$$
\ln \Gamma(x+1)-\left(x+\frac{1}{2}\right) \ln x+x-\ln \sqrt{2 \pi}=\sum_{m=1}^{\infty} \frac{B_{2 m}}{2 m(2 m-1) x^{2 m-1}}
$$

and

$$
\psi^{\prime}(x)=\frac{1}{x}+\frac{1}{2 x^{2}}+\sum_{m=1}^{\infty} \frac{B_{2 m}}{x^{2 m+1}}=\sum_{m=1}^{\infty} \frac{B_{m-1}}{x^{m}},
$$

see $[1$, p. $257,6.1 .40]$ and $[1$, p. $260,6.4 .11]$, figures out

$$
h(x)=\sum_{m=1}^{\infty} \frac{B_{2 m}}{2 m(2 m-1) x^{2 m-1}}-\sum_{m=1}^{\infty} \frac{B_{m-1}}{12\left(x+\frac{1}{2}\right)^{m}},
$$

where $B_{k}$ for $k \geq 0$ denote Bernoulli numbers which may be generated by

$$
\frac{z}{e^{z}-1}=\sum_{k=0}^{\infty} B_{k} \frac{z^{k}}{k !}=1-\frac{z}{2}+\sum_{k=1}^{\infty} B_{2 k} \frac{z^{2 k}}{(2 k) !}, \quad|z|<2 \pi .
$$

Making use of

$$
\begin{aligned}
& \sum_{k=1}^{m} \frac{a_{k}}{\left(x+\frac{1}{2}\right)^{k}}=\sum_{k=1}^{m} a_{k}\left(1+\frac{1}{2 x}\right)^{-k} \frac{1}{x^{k}} \\
&=\sum_{k=1}^{m} a_{k}\left[\sum_{i=0}^{\infty}\left(\begin{array}{c}
-k \\
i
\end{array}\right) \frac{1}{2^{i} x^{i}}\right] \frac{1}{x^{k}}=\sum_{k=1}^{m} \sum_{i=0}^{\infty} \frac{a_{k}}{2^{i}}\left(\begin{array}{c}
-k \\
i
\end{array}\right) \frac{1}{x^{k+i}}
\end{aligned}
$$

in (3.2), where $a_{k}$ is any sequence and

$$
\left(\begin{array}{c}
-k \\
i
\end{array}\right)=\frac{1}{i !} \prod_{\ell=0}^{i-1}(-k-\ell),
$$

we obtain that

$$
h(x)=\frac{1}{240} \frac{1}{x^{3}}-\frac{11}{6720} \frac{1}{x^{5}}+\frac{107}{80640} \frac{1}{x^{7}}-\frac{2911}{1520640} \frac{1}{x^{9}}+O\left(\frac{1}{x^{11}}\right) .
$$

The proof of Theorem 3.1 is complete.

Theorem 3.2. For every integer $x \geq 1$, we have

$$
\exp \left(\frac{1}{240 x^{3}}-\frac{11}{6720 x^{5}}\right)<\frac{e^{x} \Gamma(x+1)}{x^{x} \sqrt{2 \pi x} \exp \left(\frac{1}{12} \psi^{\prime}\left(x+\frac{1}{2}\right)\right)}<\exp \frac{1}{240 x^{3}} .
$$

Proof. Let $f(x)$ and $g(x)$ for $x \in[1, \infty)$ be defined by (2.7) and (2.8) respectively. Making use of inequalities

$$
\begin{aligned}
\frac{1}{12 x}-\frac{1}{360 x^{3}}+\frac{1}{1260 x^{5}} & <\ln \Gamma(x+1)-\left(x+\frac{1}{2}\right) \ln x+x-\frac{1}{2} \ln (2 \pi) \\
& <\frac{1}{12 x}-\frac{1}{360 x^{3}}+\frac{1}{1260 x^{5}}-\frac{1}{1680 x^{7}}
\end{aligned}
$$


and

$$
\frac{1}{x}+\frac{1}{2 x^{2}}+\frac{1}{6 x^{3}}-\frac{1}{30 x^{5}}<\psi^{\prime}(x)<\frac{1}{x}+\frac{1}{2 x^{2}}+\frac{1}{6 x^{3}}-\frac{1}{30 x^{5}}+\frac{1}{42 x^{7}},
$$

which may be deduced from [2, Theorem 2 and Corollary 1], finds that $f(x)<a(x)$ and $g(x)>b(x)$, where

$$
\begin{aligned}
a(x)= & \frac{1}{12 x}-\frac{1}{360 x^{3}}+\frac{1}{1260 x^{5}}-\frac{1}{12}\left[\frac{1}{x+\frac{1}{2}}+\frac{1}{2\left(x+\frac{1}{2}\right)^{2}}\right. \\
& \left.+\frac{1}{6\left(x+\frac{1}{2}\right)^{3}}-\frac{1}{30\left(x+\frac{1}{2}\right)^{5}}\right]-\frac{1}{240 x^{3}} \\
= & -\frac{A(x-1)}{5040 x^{5}(2 x+1)^{5}} \\
< & 0, \\
b(x)= & \frac{1}{12 x}-\frac{1}{360 x^{3}}+\frac{1}{1260 x^{5}}-\frac{1}{1680 x^{7}}-\frac{1}{12}\left[\frac{1}{x+\frac{1}{2}}+\frac{1}{2\left(x+\frac{1}{2}\right)^{2}}\right. \\
& \left.+\frac{1}{6\left(x+\frac{1}{2}\right)^{3}}-\frac{1}{30\left(x+\frac{1}{2}\right)^{5}}+\frac{1}{42\left(x+\frac{1}{2}\right)^{7}}\right]-\frac{1}{240 x^{3}}+\frac{11}{6720 x^{5}} \\
= & \frac{B(x-1)}{20160 x^{7}(2 x+1)^{7}} \\
> & 0, \\
A(x)= & 3760 x+6565 x^{2}+5310 x^{3}+1980 x^{4}+264 x^{5}+785 \\
B(x)= & 93268 x+263179 x^{2}+382830 x^{3} \\
& +315336 x^{4}+147504 x^{5}+35952 x^{6}+3424 x^{7}+12547 .
\end{aligned}
$$

The proof of Theorem 3.2 is thus complete.

Acknowledgements. The work of the first author was supported in part by the Romanian National Authority for Scientific Research, CNCS-UEFISCDI, under Grant No. PN-II-ID-PCE-2011-3-0087.

\section{REFERENCES}

[1] M. Abramowitz and I. A. Stegun (Eds), Handbook of Mathematical Functions with Formulas, Graphs, and Mathematical Tables, National Bureau of Standards, Applied Mathematics Series 55, 10th printing, Dover Publications, New York and Washington, 1972. 2, 4

[2] S. Koumandos, Remarks on some completely monotonic functions, J. Math. Anal. Appl. 324 (2006), no. 2, 1458-1461; Available online at http://dx.doi.org/10.1016/j.jmaa.2005.12. 017. 5

[3] D. S. Mitrinović, J. E. Pečarić, and A. M. Fink, Classical and New Inequalities in Analysis, Kluwer Academic Publishers, 1993. 1

[4] C. Mortici, A quicker convergence toward the $\gamma$ constant with the logarithm term involving the constant e, Carpathian J. Math. 26 (2010), no. 1, 86-91. 2

[5] F. Qi, A completely monotonic function involving the gamma and tri-gamma functions, Appl. Math. Inf. Sci. (2014), in press. 1

[6] F. Qi, A completely monotonic function involving the gamma and tri-gamma functions, available online at http://arxiv.org/abs/1307.5407. 1

[7] F. Qi, Bounds for the ratio of two gamma functions, J. Inequal. Appl. 2010 (2010), Article ID 493058, 84 pages; Available online at http://dx.doi.org/10.1155/2010/493058. 2

[8] F. Qi and Q.-M. Luo, Bounds for the ratio of two gamma functions: from Wendel's asymptotic relation to Elezović-Giordano-Pečarić's theorem, J. Inequal. Appl. 2013, 2013:542, 20 pages; Available online at http://dx.doi.org/10.1186/1029-242X-2013-542. 2 
C. MORTICI AND F. QI

[9] F. Qi and Q.-M. Luo, Bounds for the ratio of two gamma functions-From Wendel's and related inequalities to logarithmically completely monotonic functions, Banach J. Math. Anal. 6 (2012), no. 2, 132-158. 2

[10] F. Qi and Q.-M. Luo, Complete monotonicity of a function involving the gamma function and applications, Period. Math. Hungar. ?? (2014), in press; Available online at http: //dx.doi.org/10.1007/???. 2

[11] F. Qi and C. Mortici, Some best approximation formulas and inequalities for Wallis ratio, available online at http://arxiv.org/abs/1312.3782. 2

[12] H. Şevli and N. Batır, Complete monotonicity results for some functions involving the gamma and polygamma functions, Math. Comput. Modelling 53 (2011), 1771-1775; Available online at http://dx.doi.org/10.1016/j.mcm.2010.12.055. 1

[13] D. V. Widder, The Laplace Transform, Princeton University Press, Princeton, 1946. 1

(Mortici) Department of Mathematics, Valahia University of TÂrgovişte, Bd. Uniril 18, 130082 TÂrgovişte, Romania

E-mail address: cmortici@valahia.ro

$U R L$ : http://www.cristinelmortici.ro

(Qi) College of Mathematics, Inner Mongolia University for Nationalities, Tongliao City, Inner Mongolia Autonomous Region, 028043, China; Department of Mathematics, College of Science, Tianjin Polytechnic University, Tianjin City, 300387, China; Institute of Mathematics, Henan Polytechnic University, Jiaozuo City, Henan Province, 454010, CHINA

E-mail address: qifeng618@gmail.com, qifeng618@hotmail.com, qifeng618@qq.com

$U R L$ : http://qifeng618. wordpress .com 